\title{
Cinematic rendering in vascular imaging: Abdominal aortic aneurysm (AAA) treated with fenestrated endovascular aortic repair (FEVAR)
}

\author{
Georg Hagleitner ${ }^{1 *}$, Peter Pichler ${ }^{1}$, Paul Kiblböck ${ }^{1}$ and Franz A Fellner ${ }^{1,2}$ \\ ${ }^{1}$ Central Radiology Institute, Kepler University Hospital, Medical Faculty of the Johannes Kepler University, Linz, Austria \\ ${ }^{2}$ Medical Faculty of the Friedrich-Alexander-University of Erlangen-Nürnberg, Erlangen, Germany
}

\begin{abstract}
Cinematic rendering (CR) of cross-sectional imaging data is a novel technique of post-processing cross-sectional imaing data. Through photorealistic CR images it is now possible to deliver a better understanding anatomy and pathoanatomy for medical professionals, students and the general public alike. Computed tomography (CT) plays an essential role in diagnosis of abdominal aortic aneurysms (AAA), planning and follow-up in fenestrated endovascular aortic repair (FEVAR). In the last few years FEVAR has emerged as a standard procedure for the treatment of AAAs involving the visceral and renal arteries. In this article, we want to discuss the possible applications for CR images in a case with AAA treated with FEVAR.
\end{abstract}

\section{Introduction}

AAAs are defined as a widening of the abdominal aorta above $3,0 \mathrm{~cm}$ or more and occur with a prevalence of $4-7,2 \%$ in screening studies [1]. Highest prevalence is found in male smokers between 50 and 79 years. Important risk factors are advanced age, male gender, hypertension, hypercholesterinemia, smoking, atherosclerosis, other aneurysms, coronary or cerebrovascular disease, positive family history, and certain genetic factors [1,2]. Unruptured and uncomplicated AAAs are usually clinically silent. In some cases, a pulsatile abdominal mass can be assessed on clinical examination [2]. Ultrasound screening is recommended in populations with increased risk for the development of an AAA [1-3]. Since the 12-month AAA rupture risk is $<1 \%$ in aneurysm diameters $<50 \mathrm{~mm}$, aneurysm repair should be considered in patients with an AAA diameter $>50 \mathrm{~mm}$ [1]. Cross-sectional imaging, especially multi-phase CT-angiography, is considered as a gold standard for the preoperative assessment of AAAs [1-3].

In the last few years, FEVAR has been established as an important option for the treatment of complex AAAs with involvement of the celiac trunk, the superior mesenteric artery and the renal arteries [3]. In this procedure, endovascular repair also includes the implantation of stent grafts in the involved visceral and/or renal vessels through so-called fenestrations in the main body stent graft. Due to frequent anatomic variants regarding the renal arteries, it is sometimes necessary to plan more than four fenestrations, thus the stent graft main body usually has to be designed individually for each patient. An alternative to FEVAR is the snorkel/chimney technique, where the perfusion of the visceral and renal vessels is maintained through stentgrafts laying alongside with an unfenestrated main graft tube $[4,5]$. In the postoperative follow-up after FEVAR, multiphase CT-angiography is necessary within the first month after the procedure and in 12-month-intervals to detect early and late complications [3]. The most important complication is the reperfusion and possible extension of the aneurysmal sac through an endoleak [5].
Especially in the demonstration of vascular pathologies, anatomical and pathoanatomical particularities can be challenging to understand for non-radiologists based on cross-sectional images alone and could be even more difficult for non-vascular experts, such as students or medical laypersons [6-8]. Thus, 3D reconstructions of cross-sectional images might be helpful in teaching and illustrating pathoanatomical relations and posttherapeutic particularities. In this field, cinematic rendering has emerged as a useful technology to provide photorealistic 3D images [6-9]. In contrast to maximum intensity projections (MIP) and volume rendering (VR), CR images are based on the calculation of complex light and shadow models, used in animation trick film for example. Thus, CR allows a more realistic illustration of complex anatomic regions and pathoanatomic relations $[6,9]$.

\section{Case report}

We present a case of a 78-year old male patient with an AAA involving the celiac trunk, the superior mesenteric artery and the renal vessels. As a norm variant doubled renal vessels on both sides were present. Regarding vascular medical history, a crossover bypass was made after an occlusion of the right common iliac artery. After discussion in the interdisciplinary vascular board, indication for endovascular repair was made. We decided to implant the fenestrated stentgraft through a single access via the left common femoral artery.

${ }^{\star}$ Correspondence to: Georg Hagleitner, Central Radiology Institute, Kepler University Hospital, Medical Faculty of the Johannes Kepler University, Linz, Austria, E-mail: georg.hagleitner@kepleruniklinikum.at

Key words: cinematic rendering, virtual pathoanatomy, interventional radiology, abdominal aortic aneurysm (AAA), fenestrated endovascular aortic repair (FEVAR)

Received: October 12, 2020; Accepted: October 22, 2020; Published: October 25,2020 




Figure 1. Preoperative coronal MIP image from CT data shows the partially thrombosed aneurymatic sac. Occluded right iliac axis with previously implanted stent graft



Figure 2. Preoperative Volume Rendering image



Figure 3. Preoperative Cinematic Rendering from CT data. Clear demonstration of the abdominal aortic aneurysm, the occlusion of the right iliac axis and the crossover bypass, and their anatomic relations



Figure 4. Preoperative detail from Cinematic Rendering. Clear illustration of the doubled renal arteries on both sides

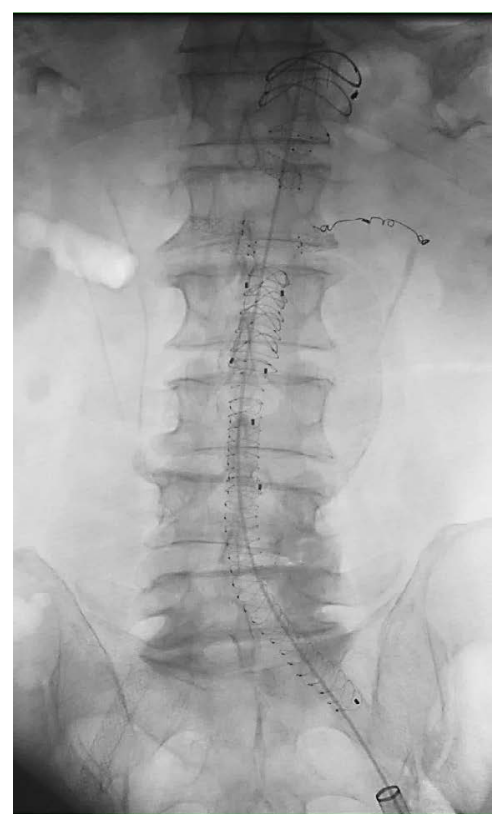

Figure 5. Intraoperative $x$-ray image at the end of the procedure. Main stent graft, aortouniiliacal prosthesis, sidebranches in situ. Note the coil in the small-caliber left renal artery

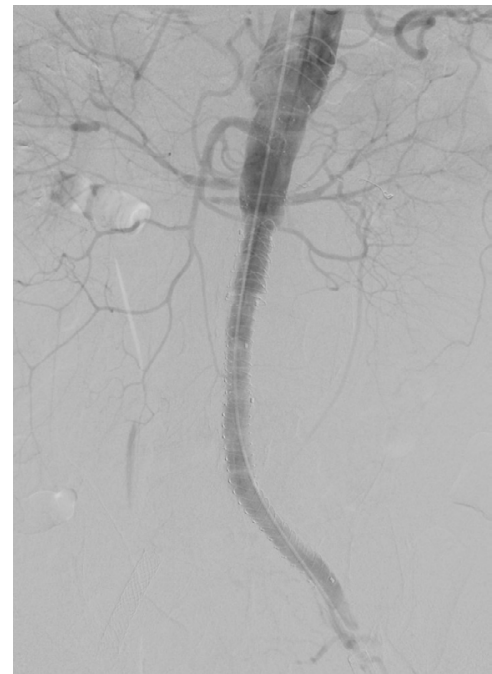

Figure 6. Final angiogram at the end of the procedure, all maintained mesenteric vessels show good contrast flow 




Figure 7. Intraoperative detail from the mesenteric root with the stent graft and the fenestrated main body. Coil in small calibrated accessory renal artery on the left

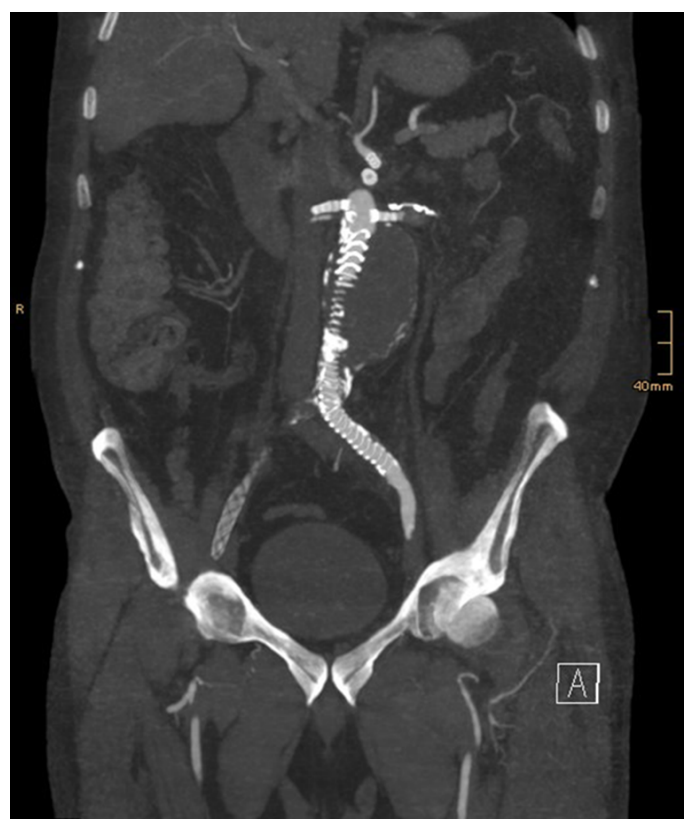

Figure 8. Coronal MIP image shows successful exclusion of the aneurysmatic sac. However, the whole prosthesis is not visible in one image



Figure 9. Postoperative Volume Rendering image



Figure 10. Cinematic Rendering image. Photorealistic depiction of the stent graft and its sidebranches, the coil in the left small calibrated accessory renal artery, and the maintained blood flow in the crossover bypass. Note the missing enhancement of the lower left kidney pole after coil embolization of the lower pole artery 


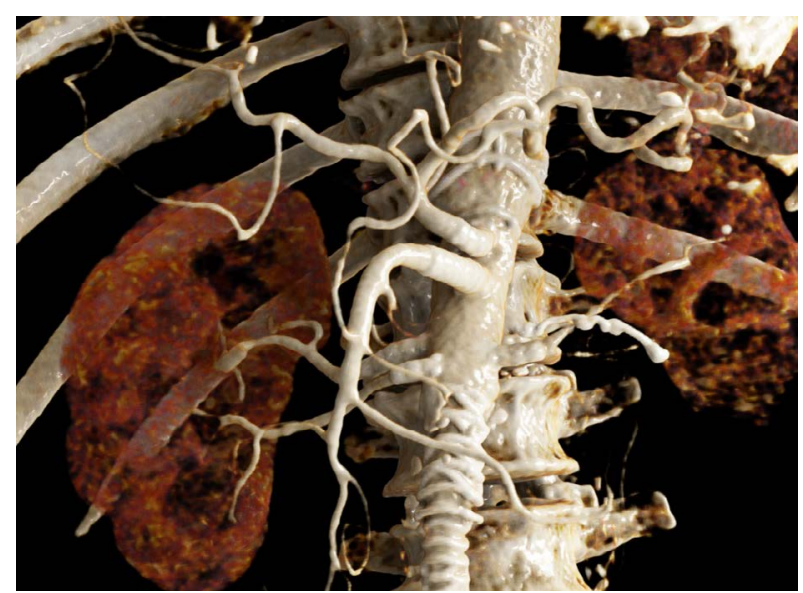

Figure 11. Detailed Cinematic Rendering. Photorealistic illustration of the mesenteric root and the maintained renal arteries

After surgical preparation of the left groin, a small-caliber renal artery on the left side was embolized with detachable coils (Interlock, Boston Scientific). After insertion of the individually designed fenestrated main graft tube (Anaconda, Terumo), blood flow to the remaining five visceral vessels - celiac trunk, superior mesenteric artery, left renal artery, superior and inferior doubled right renal artery - was maintained by insertion of covered stent grafts (Viabahn, Gore) through the fenestrations. Finally, an aortouniiliacal prosthesis was implanted (Anaconda, Terumo).

In the final angiogram and in the first postoperative CT-angiogram no early complications or endoleak were found. The peri- and postoperative course was uneventful. The patient was discharged in good general condition on the $7^{\text {th }}$ postoperative day.

\section{Discussion}

In aortic and vascular diseases, it can be challenging to understand pre- and posttherapeutic anatomical and pathoanatomical particularities, especially for non-vascular experts, medical students and/or medical lay persons [6-8].

Digital post-processing techniques, such as multiplanar reconstructions (MPR), MIPs or VR are long established tools for easier assessment and understanding of complex medical problems. In comparison to VR, CR creates photorealistic images based on complex light and shadow models $[6,9]$. This could be useful in interdisciplinary boards to facilitate understanding complex pathologies. Through the development of new reconstruction algorithms of cross-sectional imaging data such as $\mathrm{CR}$, it is now possible to present complex pathologies to a broader audience and provide an easier understanding of these, often challenging problems [6-9]. Since the establishment of endovascular procedures as a suitable option for aortic repair, it might be useful to use photorealistic reconstructions such as CR images to demonstrate pre- and posttherapeutic particularities in interdisciplinary conferences, in the planning phase with industrial partners, in the informed consent discussion with the patient, and for educational purposes with medical students, health-care students or medical laypersons [3,6-8,10-12].

\section{Conclusion}

FEVAR is an established procedure for endovascular repair of AAAs involving the visceral vessels. CR can be helpful to illustrate anatomical and pathoanatomical particularities and facilitate understanding of complex problems for medical professionals, medical and health-care students and medical lay persons.

\section{Conflicts of interest}

The authors declare that they have no conflicts of interest.

\section{Funding statement}

There was no financial support for research and publication of this article.

\section{References}

1. Moll FL, Powell JT, Fraedrich G, Verzini F, Haulon S, et al. (2011) Management of abdominal aortic aneurysms - clinical practical guidelines of the european society for vascular surgery. Eur J Vasc Endovasc Surg 41: S1-S58.

2. Erbel R, Aboyans V, Boileau C, Bossone E, Di Bartolomeo R, Eggebrecht H, et al (2014) 2014 ESC Guidelines on the diagnosis and treatment of aortic diseases. European Heart Journal 35: 2873-2926. [Crossref]

3. Chaikof EL, Dalman RL, Eskandari MK, Jackson BM, Lee WA, et al. (2018) The Society for Vascular Surgery practice guidelines on the care of patients with an abdominal aortic aneurysm. J Vasc Surg 67: 2-77. [Crossref]

4. Swerdlow NJ, Wu WW, Schermerhorn ML (2019) Open and Endovascular Management of Aortic Aneurysms. Circ Res 124: 647-661. [Crossref]

5. Wanhainen A, Verzini F, van Herzeele I, Allaire E, Bown M, et al. (2019) European Society for Vascular Surgery (ESVS) 2019 Clinical Practice Guidelines on the Management of Abdominal Aorto-iliac Artery Aneurysms. Eur J Vasc Endovasc Surg 57: 8-93

6. Fellner FA (2016) Introducing Cinematic Rendering: A novel technique for postprocessing medical imaging data. J Biomedical Science and Engineering 9: 170175.

7. Fellner FA, Berger F, Fellner C, Kremer C, Hortner H, Stocker G (2016) Volume Rendering of medical imaging data for the general public. J Health Med Informat 7: 213.

8. Binder J, Krautz C, Engel K, Grützmann R, Fellner FA, et al. (2019) Leveraging medical imaging for medical education - a cinematic rendering-featured lecture. Ann Anat 222: 159-165. [Crossref]

9. Elshafei M, Binder J, Baecker J, Brunner M (2019) Comparison of Cinematic Rendering and Computed Tomography for Speed and Comprehension of Surgical Anatomy. JAMA Surg 154: 738-744. [Crossref]

10. Fellner FA, Engel K, Kremer C (2017) Virtual Anatomy: The dissecting theatre of the future - implementation of Cinematic Rendering in a large $8 \mathrm{~K}$ high-resolution projection environment. J Biomedical Science and Engineering 10: 367-375.

11. Hofko M, Sonnberger M, Fellner FA (2020) Cerebral dural arterio-venous fistula - Par I: Virtual anatomy and pathoanatomy in CT and MR imaging. Trauma Emerg Care 5.

12. Hagleitner G, Pichler P, Fellner FA (2020) Understanding vascular pathoanatomy: Cinematic Rendering of an abdominal aortic aneurysm treated with chimney endovascular aortic repair. Glob Imaging Insights 5.

Copyright: (C2020 Hagleitner G. This is an open-access article distributed under the terms of the Creative Commons Attribution License, which permits unrestricted use, distribution, and reproduction in any medium, provided the original author and source are credited. 